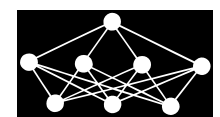

\title{
AN EFFICIENT METHOD FOR SURFACE RECONSTRUCTION BASED ON LOCAL COORDINATE SYSTEM TRANSFORM AND PARTITION OF UNITY
}

\author{
Z. Zhou, Y. Fu J. Zhao
}

\begin{abstract}
Radial basis function (RBF) has been extensively applied for surface reconstruction from scattered 3D point data due to its strong ability of approximation. However, additional information, such as off-surface points, are usually required to be appended into constraints for determining the parameters, which apparently increases the computation cost and data unreliability. To avoid adding additional off surface point constraints, a novel surface reconstruction approach based on local coordinate system transform and partition of unity is proposed in this paper. Firstly, the explicit RBF functions are constructed to approximate the local surface patches, and then it is transformed into an equivalent implicit surface reconstruction form by local system coordinate transformation. Compared with the local implicit surface approximation, the proposed local explicit surface approximation method is capable of avoiding trivial solution occurred in RBF approximating, and does not increase the scale of data solution. A number of comparison experiments of the proposed method with the traditional RBF-based method and the multi-level partition of unity (MPU) method are carried out on some kinds of large dataset, non-uniformity dataset, noisy dataset. The experimental results illustrate that the proposed method is robust and effective in dealing with large-scale point clouds surface reconstruction.
\end{abstract}

Key words: surface reconstruction, radial basis function, partition of unity, local coordinate system transform

Received: January 5, 2019

DOI: $10.14311 / N N W .2020 .30 .012$

Revised and accepted: June 30, 2020

\section{Introduction}

Along with the quick development of 3D scan technology, millions of contour points on the surface of object can be obtained. These points are often used to reconstruct the watertight surface in the areas of geometry processing, scientific visualization,

\footnotetext{
*Zhenghua Zhou - Corresponding author; College of Sciences, China Jiliang University, Hangzhou, Zhejiang Province, China, 310018, E-mail: zzh@cjlu.edu.cn

†Yanqing Fu; Jianwei Zhao ; College of Sciences, China Jiliang University, Zhejiang, Hangzhou, China, 310018, E-mail: 403375883@qq.com, zhaojw@cjlu.edu.cn
} 
and their applications $[6,9,14,17,23]$. In the last two decades, surface reconstruction has become one of the most significant topics in computer graphics, and substantial numbers of techniques have been developed. But it is still a challenging task for the people to build a desired surface. The main reason is that the obtained data are usually huge, non-uniform, full of noises, and even with some holes due to the inaccessibility of scan. Generally, a desirable method for surface reconstruction should include a good reconstruction accuracy, a fast reconstruction speed, a low memory cost, and a good faith to some points with the low quality as mentioned above.

According to the representation of the reconstructed surface, the existing methods for surface reconstruction are mainly classified into two categories: explicit method and implicit method. The explicit method aims to find an explicit continuous function to interpolate or approximate those scanned points [8,25], while the implicit method seeks to build an implicit continuous function whose zero-set is the reconstructed surface to interpolate or approximate those points [5]. Specifically, let $\mathcal{P}=\left\{p_{i}\right\}_{i=1}^{N} \subseteq \mathbb{R}^{3}$ be the set of scanned points associated with their corresponding unit normal vectors $\mathcal{N}=\left\{n_{i}\right\}_{i=1}^{N} \subseteq \mathbb{R}^{3}$, where $\mathcal{N}$ forecasts the orientation of the target surface $\mathcal{S}$ and it can be directly obtained by some scanner device or estimated with principle component analysis (PCA) or the local least-square fitting. Then the traditional explicit methods interpolate or approximate the target surface $\mathcal{S}$ by means of a continuous bivariate function or a series of simple parametric surfaces, while the implicit methods aim at constructing a continuous function $f: \mathbb{R}^{3} \rightarrow \mathbb{R}$ such that its zero-set $\left\{p \in \mathbb{R}^{3}: f(p)=0\right\}$ can approximate the target surface $\mathcal{S}$.

Due to the complexity and closure of the surface used for representing object, the implicit method can obviously construct a closer surface than the explicit method does. However, the existing implicit methods usually need to be appended with some additional constrains such as adding off-surface points to help determine the parameters, which increases the memory cost, computational complexity and data noises dramatically. Hermite implicit method replaces the off-surface points with the normal vectors' approximators in the additional constraint equations [16,22], so its computational complexity sometimes is still unacceptable for the large dataset. Therefore, a natural question is: Is there any implicit method that does not need off-surface points and has a satisfied computational complexity? This paper gives an affirmative answer for this question.

In this paper, we will propose a novel implicit method for the 3D surface reconstruction without adding other additional constrains by using the local coordinate system transform. First, similar to the traditional local RBF-based method, we partition the large data domain into some small subdomains. It is noted that we need ensure that each local surface sheet has an explicit expression in some new coordinate system. Then in order to avoid adding additional constraints and determine the parameters in the traditional local RBF-based method, we get a non-homogeneous linear system with a transformation between the original scanning coordinate system and the new coordinate system. Finally, the global function is obtained by blending each local approximation with some local weighed functions [19]. Therefore, our proposed method is a mixture of RBF-based and multi-level partition of unity (MPU) based method. On one hand, our proposed 
method can be considered as an improved RBF-based method, but it can do more than the traditional RBF-based method does. The classical implicit RBF-based method needs to add some off-surface data, while our method avoids this process by transforming the homogeneous linear system into a non-homogeneous case with a transformation of the coordinate system. On the other hand, our proposed method can be regarded as the RBF-based MPU method. It firstly divides the dataset into a series of cells with a fixed size, then in each local coordinate system, local surface represented as an explicit a bivariate function is reconstructed. The bivariate function is taken to be the RBF.

The main contribution of the paper can be summarized as follows:

- The local coordinate system transform and partition of unity are combined to perform surface reconstruction, which does not need extra off-set points.

- The proposed approach can quickly and accurately reconstruct complex topological models.

- The bivariate function in the form of radial basis function used for approximating the local surface patches is robust to noisy data and non-uniformity datasets.

The rest of the paper is organized as follows. We first review the previous work of the surface reconstruction in Section 2. Then, we give a specific processes of our proposed method for the surface reconstruction in Section 3. In Section 4 , experimental results on different shape datasets with comparison to existing methods are given. Finally, we conclude the paper in Section 5.

\section{Related work}

Up to now, a lot of approaches for surface reconstruction have been proposed. According to the processing techniques, these approaches are roughly classified into three groups: Delaunay triangulation (or Voronoi diagram)-based methods, parametric methods, and implicit methods.

Delaunay triangulation-based methods. Delaunay triangulation based methods generally construct the surface by exploiting the structures, such as Delaunay tetrahedral and Voronoi diagrams. That is, they usually interpolate all or most of the points by means of creating a triangle mesh. Some representative methods of this group are $\alpha$-shapes [8], crust and power crust algorithms [2], tight cocone [6], and so on. The main shortcoming of these methods is that they are usually sensitive to the noise.

Parametric methods. Parametric methods aim to build the reasonable correspondence between the pairs of locations on the boundaries of the two shapes. The popular representative methods are B-spline method [10] and non-uniform rational B-spline (NURBS) surface [21]. These methods are typically faster and require less memory than Delaunay triangulation-based methods because they operate on a lower dimensional representation of an object. However, their expression ability is limited by the parametric functions.

Implicit methods. Different from the above two methods, the goal of implicit methods is to find a continuous function and uses its zero isosurface to fit those 
discrete points. Most existing implicit methods for constructing the continuous function are virtually determined by the linear combination of the basis functions such as signed distance function [11,25], RBF [5,16,20], local implicit functions [19], moving least square approximation [1], indicator functions [12], and so on. Among these methods, the local RBF-based method [24] and MPU method [19] present a good performances in dealing with the large dataset. But they also have deficiency. For example, RBF-based method is powerful for the complex topology model and robust to the noises, but it requires some additional information, such as off-surface points, to determine the functions, which is apparently not reliable for the cases with noises or some closed sheets. While for the MPU method, although it can reconstruct the sharp feature for the non-uniformity or large datasets efficiently with the local quadric polynomial and the partition of unity, it is very sensitive to the noise because of the rigidity of the quadric polynomial.

\section{The proposed surface reconstruction method}

In this section, we will propose a new implicit surface reconstruction method based on the partition of unity (PU) and RBF for the glocal surface reconstruction. The proposed method is composed of three stages as follows: Firstly, for the given scan data, we divide the global domain into several local sub-domains based on the adaptive Octree-based subdivision method. Then we reconstruct the local surface with the proposed implicit method. Finally, the global surface is obtained by the PU method. A block schematic diagram of the proposed method is shown in Fig. 1.

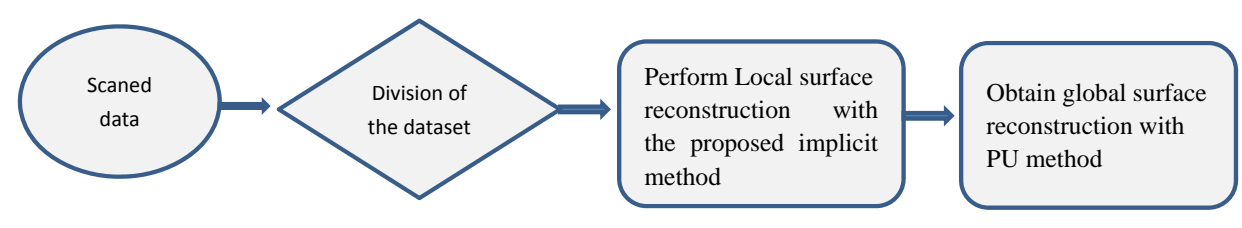

Fig. 1 Diagram of the proposed method for surface reconstruction.

\subsection{Division of the dataset}

Due to the complexity of the surface and the huge volume of the scanned data, we intend to reconstruct the global surface by means of reconstructing each simple local surface. Therefore, the first work is to divide the global domain into several local domains. Here, our proposed adaptive Octree-based subdivision method in [26], which is developed based on [19], is employed to achieve the data division task.

Let $S_{0}$ be the minimum bounding box which can contain the scanned dataset $\left\{\left(p_{i}, n_{i}\right)\right\}_{i=1}^{N}$, where $p_{i} \in R^{3}$ is the scanned position point and $n_{i}$ is its corresponding unit normal vector. It is assumed that $N_{\min }$ and $N_{\max }$ are the minimum and maximum number of the points ensuring that each sub-domain allows to contain, respectively. If the number of points in the domain $S_{0}$ is larger than $N_{\max }$, then 
divide $S_{0}$ into eight overlapping sub-domains. For these eight sub-domains, if their numbers of points are larger than $N_{\max }$, then we continue to divide these obtained sub-domains. The whole subdivision algorithm for the scanned dataset is detailed as follows:

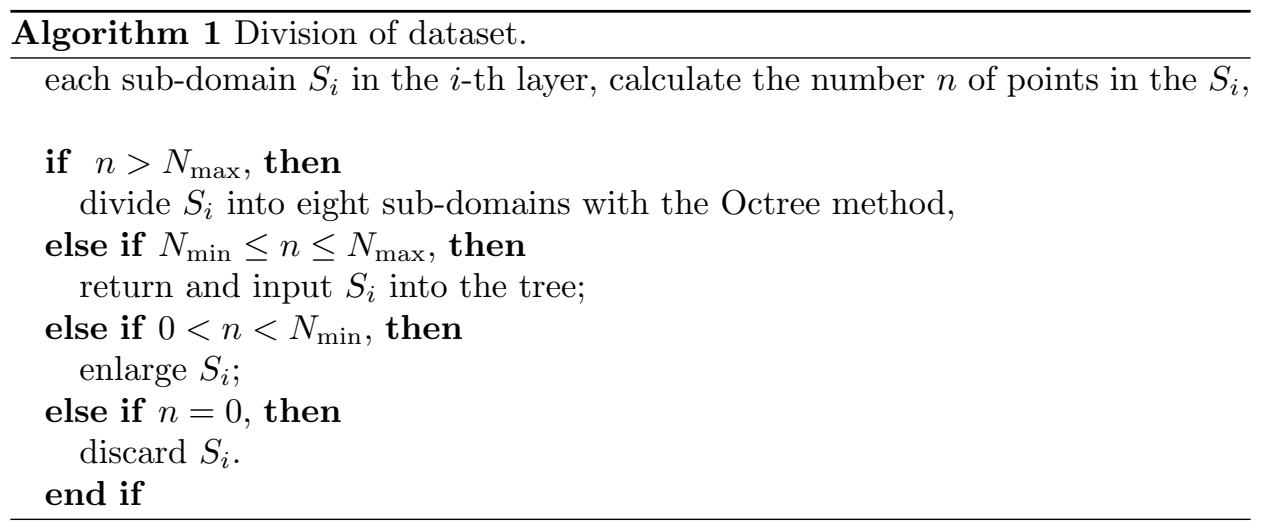

In the next step of our proposed method, we assume that each local surface to be reconstructed has an explicit expression. Therefore, we use $N_{\min }$ and $N_{\max }$ to adjust the structure of each local surface.

\subsection{The proposed method for the local surface reconstruction}

For each divided sub-domain $S_{j}$ that contains point set $\left\{\left(p_{i}, n_{i}\right)\right\}_{i=1}^{N_{j}}$ with the division algorithm, the traditional implicit method aims to seek a smooth function $f_{j}$ : $\mathbb{R}^{3} \rightarrow \mathbb{R}$ from some smooth function space $\mathcal{H}$ satisfying $f_{j}\left(p_{i}\right)=0, i=1,2, \ldots, N_{j}$. According to the approximation theory, those smooth functions in $\mathcal{H}$ are usually taken as the form of RBF as follows:

$$
f_{j}(p)=\sum_{i=1}^{N_{j}} \alpha_{j i} \phi\left(p, p_{i}\right)+Q_{j}(p),
$$

where each $\alpha_{j i} \in \mathbb{R}, \phi: \mathbb{R}^{3} \times \mathbb{R}^{3} \rightarrow \mathbb{R}$ is the radial basis function, and $Q_{j}$ is the usual polynomial with one order, i.e.

$$
Q_{j}(p)=c_{j 0}+c_{j 1} x+c_{j 2} y+c_{j 3} z, \quad p=(x, y, z)^{\top} .
$$

Therefore, we need to determine those parameters for obtaining the reconstructed surface. Usually, those parameters are determined by solving the following homogeneous linear system [5]:

$$
\left(\begin{array}{cc}
\Phi & P_{j}^{\top} \\
P_{j} & 0
\end{array}\right)\left(\begin{array}{c}
\alpha_{j} \\
c_{j}
\end{array}\right)=0
$$

where $\Phi=\left[\phi_{h k}\right]_{N_{j} \times N_{j}}$ with each $\phi_{h k}=\phi\left(p_{h}, p_{k}\right), P_{j} \in \mathbb{R}^{4 \times N_{j}}$ with the $i$-th column vector being $\left(1, x_{i}, y_{i}, z_{i}\right)^{\top}, \alpha_{j}=\left(\alpha_{j 1}, \alpha_{j 2}, \ldots, \alpha_{j N_{j}}\right)^{\top}, c_{j}=\left(c_{j 0}, c_{j 1}, c_{j 2}, c_{j 3}\right)^{\top}$. 
To avoid the trivial solution of (3), many traditional methods usually introduce a number of off-surface points for increasing some constrain equations in (3). But such process of adding the off-surface points inevitably increase the scale of solving equation and the instability of system. In order to avoid adding other constraint equations, we seek to change the implicit approximation surface function into explicit bivariate surface function via transforming the homogeneous linear system into a non-homogeneous linear system. For each local surface patch, suppose that the local surface patch has the explicit expression in this coordinate plane, a local coordinate plane is established, and the projection value of the patch to the plane is taken as its local function value. Then the relation between two coordinate systems is employed to yield a nonzero vector on the right side of system (3), which needs not to add other constraint equations. In the following section, we will describe local surface reconstruction process in detail.

Suppose that the geodetic coordinate system for scanning is denoted by $O-x y z$, and the local coordinate system is represented by $P_{j}-n_{j} l_{j} m_{j}$.

According to the theory of spatial analytic geometry, there exists a unique transfer matrix $A_{j} \in \mathbb{R}^{3 \times 3}$ such that

$$
\left(l_{j}, n_{j}, m_{j}\right)^{\top}=A_{j}(x, y, z)^{\top}
$$

Namely,

$$
\left\{\begin{array}{l}
l_{j}=a_{11}^{(j)} x+a_{12}^{(j)} y+a_{13}^{(j)} z, \\
n_{j}=a_{21}^{(j)} x+a_{22}^{(j)} y+a_{23}^{(j)} z \\
m_{j}=a_{31}^{(j)} x+a_{32}^{(j)} y+a_{33}^{(j)} z
\end{array}\right.
$$

Here the matrix $A_{j}$ can be determined when the local coordinate system is chosen.Therefore, if the explicit expression for the local surface in the local coordinate system $P_{j}-n_{j} l_{j} m_{j}$ is

$$
m_{j}=h_{j}\left(l_{j}, n_{j}\right)
$$

then its implicit expression in the global geodetic coordinate system $O-x y z$ is

$$
\begin{aligned}
& a_{31}^{(j)} x+a_{32}^{(j)} y+a_{33}^{(j)} z \\
& =h_{j}\left(a_{11}^{(j)} x+a_{12}^{(j)} y+a_{13}^{(j)} z, a_{21}^{(j)} x+a_{22}^{(j)} y+a_{23}^{(j)} z\right) .
\end{aligned}
$$

Let

$$
g_{j}(x, y, z):=h_{j}\left(a_{11}^{(j)} x+a_{12}^{(j)} y+a_{13}^{(j)} z, a_{21}^{(j)} x+a_{22}^{(j)} y+a_{23}^{(j)} z\right)
$$

and

$$
\Pi_{j}(x, y, z):=a_{31}^{(j)} x+a_{32}^{(j)} y+a_{33}^{(j)} z,
$$

then the implicit expression for the local surface in the coordinate system $O-x y z$ is

$$
g_{j}(x, y, z)-\Pi_{j}(x, y, z)=0 .
$$

Let

$$
f_{j}(x, y, z):=g_{j}(x, y, z)-\Pi_{j}(x, y, z)
$$


Zhou Z., Fu Y., Zhao J.: An efficient method for surface reconstruction based on...

we use the RBF network

$$
\sum_{i=1}^{N_{j}} \alpha_{j i} \phi\left(p, p_{i}\right)+Q_{j}(p)
$$

in (1) to approximate the function $g_{j}$, then the implicit expression becomes to be

$$
\begin{aligned}
f_{j}(x, y, z) & =\sum_{i=1}^{N_{j}} \alpha_{j i} \phi\left(p, p_{i}\right) \\
& +Q_{j}(p)-\Pi_{j}(x, y, z)=0 .
\end{aligned}
$$

Now our task is to approximately determine those parameters in (13) with the scanned data. Here we obtain those parameters by solving the following nonhomogeneous linear system:

$$
\left(\begin{array}{cc}
\Phi & P_{j}^{\top} \\
P_{j} & 0
\end{array}\right)\left(\begin{array}{c}
\alpha_{j} \\
c_{j}
\end{array}\right)=\left(\begin{array}{c}
d_{j} \\
0
\end{array}\right),
$$

where $d_{j}=\left[\Pi_{j}\left(p_{1}\right), \Pi_{j}\left(p_{2}\right), \ldots, \Pi_{j}\left(p_{N_{j}}\right)\right]^{\top}$.

It is noted that the left side of the equation (14) is same as (3), but the right side is a nonzero vector. Obviously, we no longer add other off-set points. Furthermore, the expression of $f_{j}$ in (13) is still a form of RBF, which means that the function still have a pretty performance for a complex surface.

For the above non-homogeneous linear system (14), we add the $\ell_{2}$-regularization term to the optimization object function to obtain a stable solution. Therefore, parameters in (13) are finally determined by solving the following optimization problem:

$$
\min _{\beta} \sum_{i=1}^{N_{j}}\left\|f_{j}\left(p_{i}\right)\right\|_{2}^{2}+\lambda\|\beta\|_{2}^{2},
$$

where $\beta$ is the vector composed of all the parameters in the non-homogeneous linear system (14). Here we will use lower upper (LU) factorization to solve the optimization problem (15).

After explaining the idea of our proposed local surface reconstruction, we give the concrete process of determining the coefficients $a_{3 i}^{(j)}(j=1,2,3)$ of the function $\Pi_{j}$. As we know, when $m_{j}=0$, the coefficients $a_{3 i}^{(j)}(j=1,2,3)$ are the coefficients of a plane $\Pi_{j}=0$ in the original global coordinate system $O_{x y z}$. As we have divided the global domain into some sub-domains with simple structure, each local surface has a rough orientation. The orientation can be defined by those unit normal vectors of the points in each cell, so we take the following vector $\xi_{j}$

$$
\xi_{j}=\frac{n_{h}+n_{k}}{2}
$$

as the normal vector of the local plane, $n_{h}$ and $n_{k}$ are the normal vectors of the two points with the largest angle between the normal vectors in the current surface patch. 
In order to determine the local plane $\Pi_{j}$ and avoid the intersection of plane and local surface slice as much as possible, we still need another vector $\eta_{j}$ defined by

$$
\eta_{j}=c_{j}-r_{j} \xi_{j}
$$

where $c_{j}$ is the center vector of the cell, and $r_{j}$ is the radial of the bounding sphere. For the local reconstruction cell $S_{j}$ with $N_{j}$ points, the implicit function expression in (13) becomes to be

$$
\begin{aligned}
f_{j}(x, y, z) & =\sum_{i=1}^{N_{j}} \alpha_{j i} \phi\left(p, p_{i}\right) \\
& +Q_{j}(p)-\left\langle\xi_{j}, p-\eta_{j}\right\rangle=0 .
\end{aligned}
$$

\subsection{Global reconstruction surface with PU method}

In this section, we use PU method to decompose the global problem into several smaller local problems, and blend the local surfaces into a global surface with the corresponding weighted functions. For each subdomain $S_{j}$, we have obtained a local implicit function $f_{j}$ with our proposed local surface reconstruction method, $j=1,2, \ldots, M$. Let $w_{i}$ be the corresponding weighted function with $\sum_{i=1}^{M} w_{i} \equiv 1$ on the whole domain $S$. Then we can obtain the global reconstructed function $f$ as follows:

$$
f(p)=\sum_{j=1}^{M} w_{j}(p) f_{j}(p)=0 .
$$

In general, the weighted functions $\left\{w_{j}\right\}_{j=1}^{M}$ can be obtained by normalizing a set of positive functions $\left\{W_{j}\right\}_{j=1}^{M}$ as follows:

$$
w_{j}(p)=\frac{W_{j}(p)}{\sum_{i=1}^{M} W_{i}(p)} .
$$

For the implicit surface reconstruction, the global approximation function should be continuous, so every $W_{i}$ has to be continuous on the boundary of the sub-domains $S_{i}, i=1,2, \ldots, M$. According to above analysis, we summary our algorithm for surface reconstruction as follows.

\section{Experiments}

In order to evaluate the performance of our proposed method, a series of experiments are designed to show the generalization ability, effectiveness and stability of the proposed surface reconstruction model. In this part, the generalization and the stability of our proposed method is verified with various models containing different features and noises. All the experiments are implemented by Microsoft Visual Studio 2010 and all results presented in this section are run on an Intel(R) $2.40 \mathrm{GHz}$ and Xeon (R) $2.39 \mathrm{GHz}$ with 8GB RAM. The scanned database are chosen from AIM@SHAPE repository. For the visualization of the implicit surface, we adopt Bloomenthal's polygonizer method [3] to generate a polygonal mesh. The 


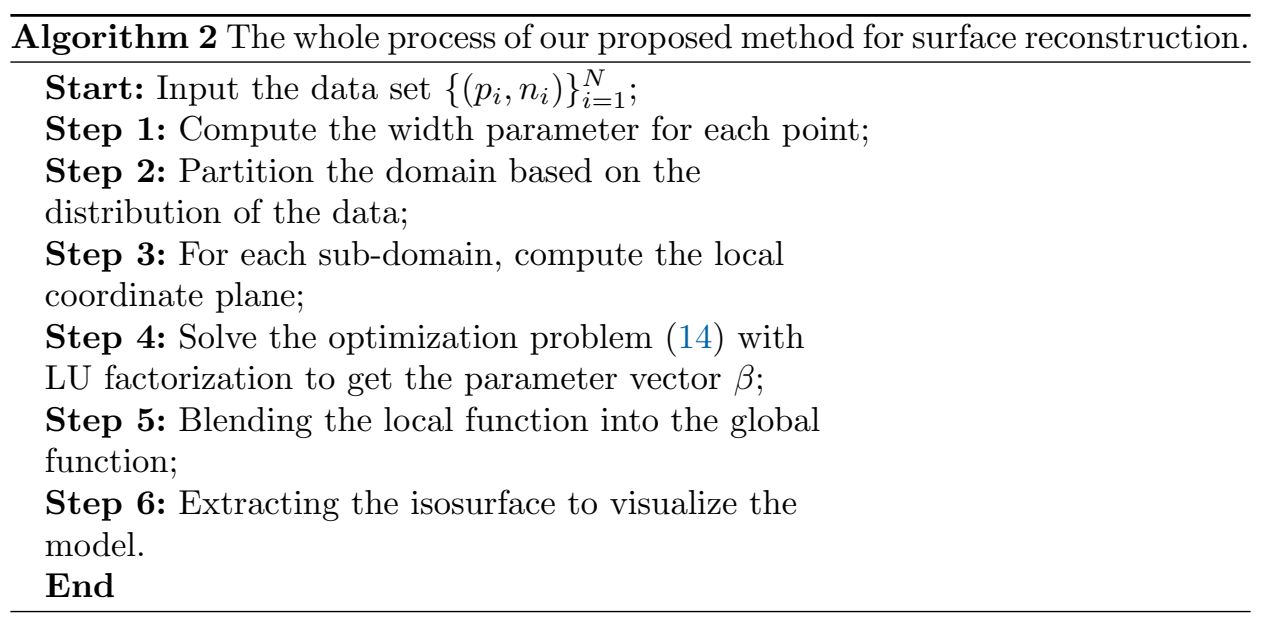

parameters in the experiments are set as follows: $N_{\min }=15, N_{\max }=30$. The radial basis function $\phi\left(p, p_{i}\right)$ is taken as $\phi\left(p, p_{i}\right)=\left(1-\left\|p-p_{i}\right\|\right)^{4}\left(4\left\|p-p_{i}\right\|+1\right)$.

\subsection{Generalization of our proposed method}

Figs. 2-5 illustrate the performance of our proposed method for different types of objects. Fig. 2 shows the reconstruction surfaces of a blade which has some planes. It is seen from Fig. 2 that our proposed method can handle the object with the non-absolute sharp edges such like the planes. On the contrary, if the scanned object has a complex local structures, such as the absolute sharp edges, our method can still achieve good performance due to polishing effect of radial basis function. Fig. 3 shows the perfect reconstruction surfaces of some complex

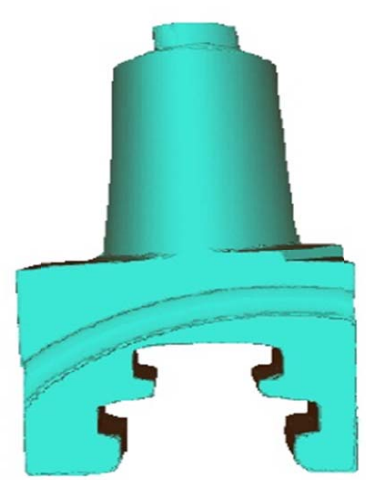

(a) Original surface of blade.

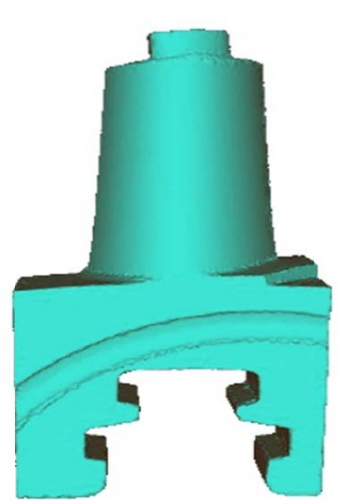

(b) Reconstructed front of blade.

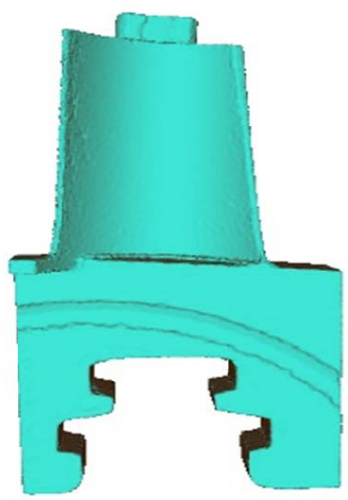

(c) Reconstructed back of blade.

Fig. 2 Reconstruction results of blade with our proposed method with 18395 points. 


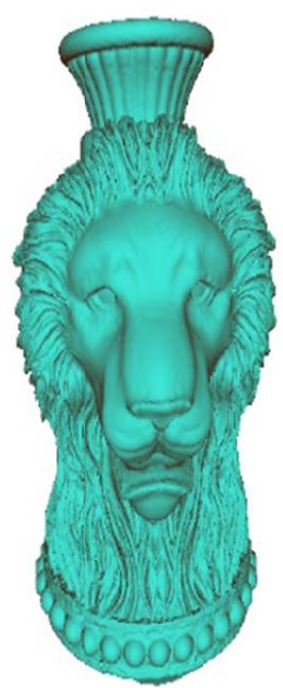

(a) Reconstructed vaselion with 200000 points.
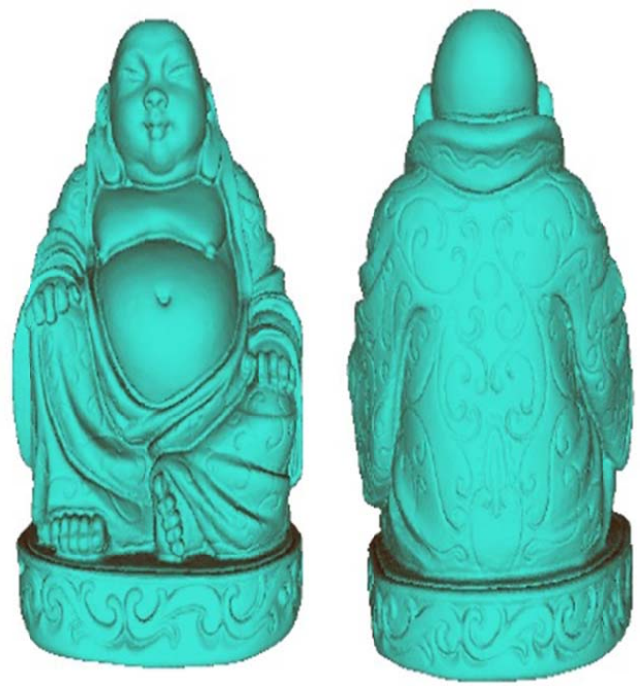

(b) Reconstructed buddha with 250000 points

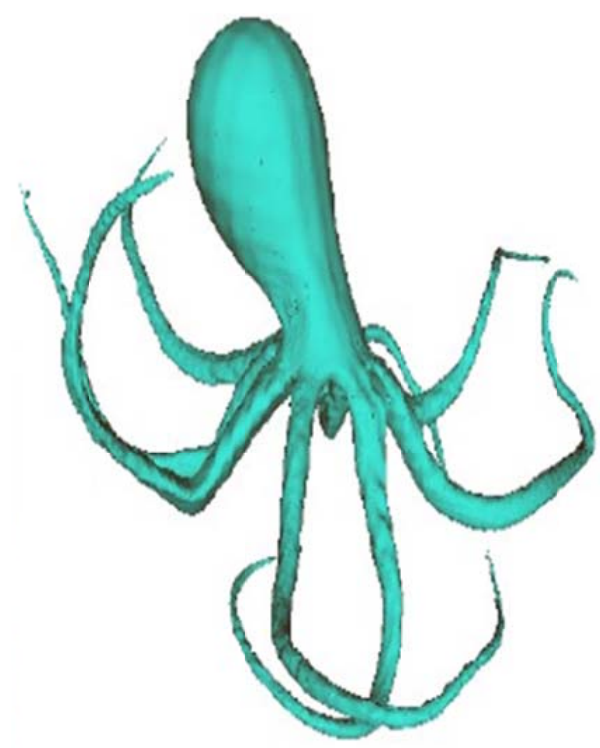

(c) Reconstructed octopus with 16944 points.

Fig. 3 Reconstruction results of some complex models with our proposed method. 
Zhou Z., Fu Y., Zhao J.: An efficient method for surface reconstruction based on...

objects with some tinny local features. Even the shallow ridges on the back of Buddha and the antennaes of octopus can be vividly described. To validate the ability of disposing large and complex point set surface, we use the raptor model with 1 million sampled points to perform the reconstruction of raptor with our proposed method as seen in Fig. 4. It is observed from Fig. 4 that the fine stripes and teeth of the raptor's head are accurately reconstructed by means of the similar size of each sub-domain. In Fig. 5, the points density on the right side of Venus is lower than that of left side as shown in Fig. 5(a). It is observed from Fig. 5(b) and Fig. 5(c) that our proposed method can accurately capture the intrinsic shape feature from these non-uniformity distribution data points.
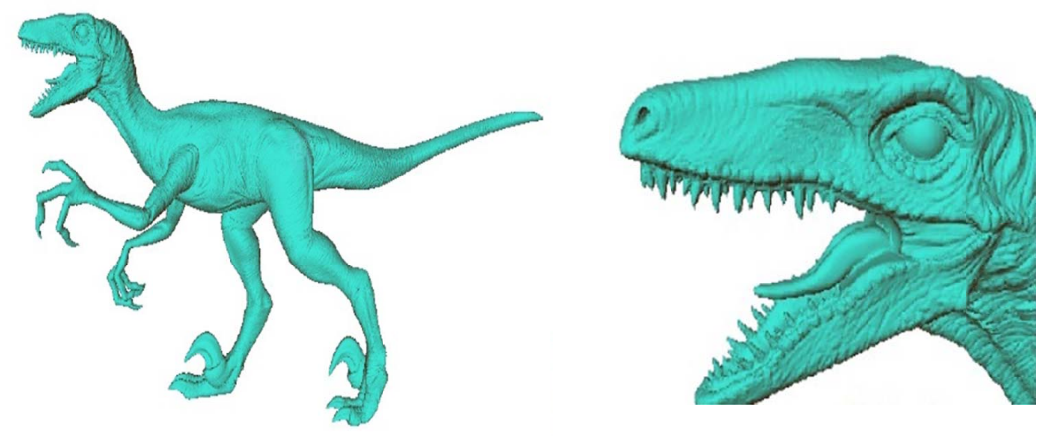

(a) Reconstructed raptor with 1000000 points (b) Reconstructed head details of raptor

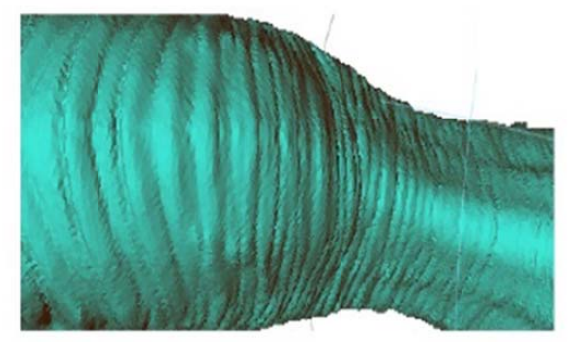

(c) Reconstructed neck ridge of raptor

Fig. 4 Reconstruction results of some complex models with our proposed method.

\subsection{Efficiency of our proposed method compared with the traditional RBF method}

To further validate the efficiency in handing large scale points surface reconstruction. Compared with the traditional RBF method for surface reconstruction, our proposed method does not need to append a large number of of off-surface points, thus reducing the computational complexity. Fig. 6 shows the comparison result of Armadillo reconstruction between our proposed method and the traditional RBF method. As shown in Fig. 6, there is no obvious visual difference between the two 


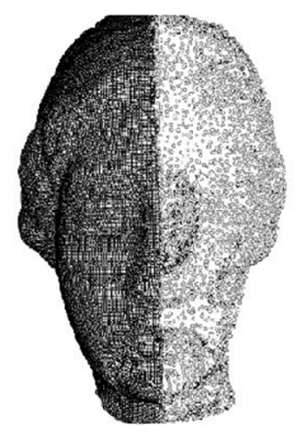

(a) Point representation of Venus.

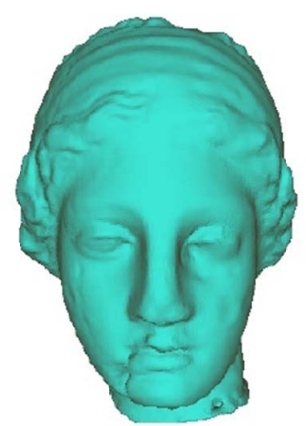

(b) Reconstructed Venus with our proposed method.

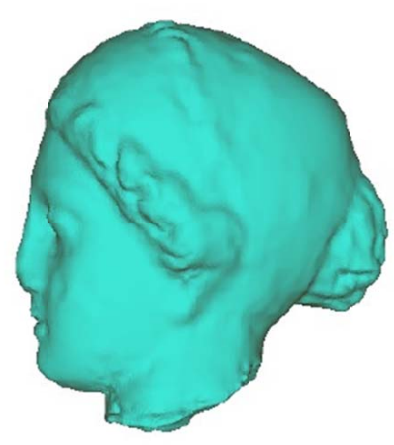

(c) Reconstruction result of Venus's side with low density points.

Fig. 5 Reconstruction result of Venus with our proposed method for non-uniformity dataset.

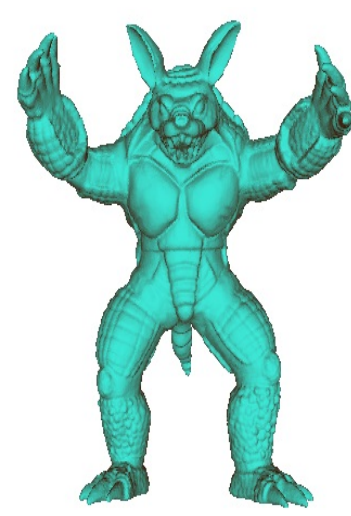

(a) Original surface of Armadillo.

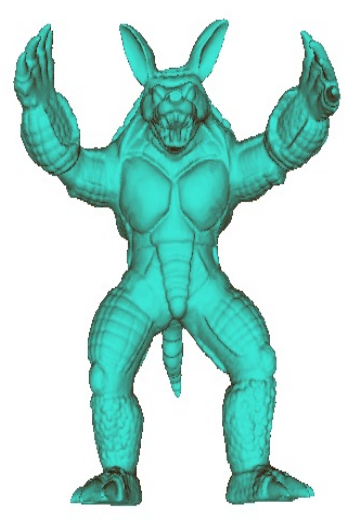

(b) Reconstructed Armadillo with our proposed method.

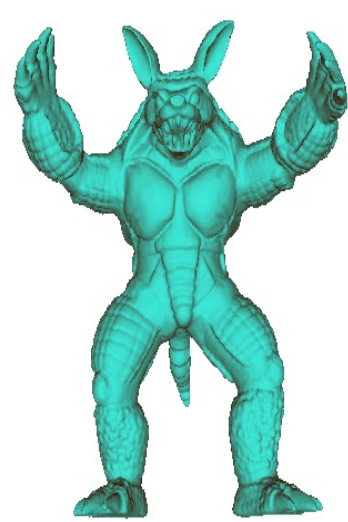

(c) Reconstructed Armadillo with traditional RBF method.

Fig. 6 Reconstructed Armadillo with our proposed method and the traditional RBF method.

methods, which indicates that our proposed method completely achieve the same reconstruction effect as the traditional RBF methods. Tab. I showns the comparison results of our proposed method with the traditional RBF method that requires off-surface points to prevent the occurrence of trivial solution.

In this comparison experiment, the number of partition cells and radial basis functions are same. In Tab. I, $N$ and $M$ are the numbers of data and the sub-domains, respectively, $T_{\text {our }}$ and $T_{\mathrm{RBF}}$ denote the computational time for the coefficients of our proposed method and the RBF method, respectively. The RMS error of our proposed method and the RBF method are denoted by $E_{\text {our }}$ and $E_{\mathrm{RBF}}$, 
Zhou Z., Fu Y., Zhao J.: An efficient method for surface reconstruction based on...

respectively. It is Observed from Tab. I that the computational time of the traditional RBF method is about twice that of our proposed method. In the meanwhile, the error of our proposed method is much less than that of the traditional RBF method.

\begin{tabular}{ccccccc}
\hline Models & $N$ & $M$ & $T_{\text {our }}(s)$ & $T_{\mathrm{RBF}}(s)$ & $E_{\text {our }}$ & $E_{\mathrm{RBF}}$ \\
\hline \multirow{4}{*}{ Armadillo } & 9679 & 3609 & 1 & 5 & $6.5 \times 10^{-5}$ & $4.6 \times 10^{-2}$ \\
& 36145 & 13173 & 6 & 19 & $4.3 \times 10^{-5}$ & $2.3 \times 10^{-2}$ \\
& 172974 & 57310 & 32 & 115 & $1.5 \times 10^{-5}$ & $7.2 \times 10^{-3}$ \\
\hline \multirow{3}{*}{ Bunny } & 12005 & 5084 & 2 & 5 & $6.8 \times 10^{-5}$ & $3.4 \times 10^{-2}$ \\
& 42399 & 20255 & 7 & 21 & $5.3 \times 10^{-5}$ & $3.4 \times 10^{-2}$ \\
& 139990 & 79430 & 30 & 68 & $2.6 \times 10^{-5}$ & $1.5 \times 10^{-2}$ \\
\hline \multirow{3}{*}{ Raptor } & 109668 & 62444 & 22 & 50 & $3.2 \times 10^{-5}$ & $3.2 \times 10^{-2}$ \\
& 413189 & 251412 & 94 & 217 & $1.2 \times 10^{-5}$ & $8.8 \times 10^{-3}$ \\
& 1000080 & 439985 & 252 & 707 & $9 \times 10^{-6}$ & $9.5 \times 10^{-3}$ \\
\hline \multirow{3}{*}{ Blade } & 7745 & 4074 & 1 & 4 & $2 \times 10^{-6}$ & $4.5 \times 10^{-2}$ \\
& 18395 & 8670 & 3 & 10 & $2 \times 10^{-6}$ & $4.2 \times 10^{-2}$ \\
& 29275 & 16798 & 7 & 18 & $1 \times 10^{-6}$ & $2.3 \times 10^{-2}$ \\
\hline
\end{tabular}

Tab. I Time (seconds) and error comparison of our proposed method with the traditional RBF method for different models.

\subsection{Stability of our proposed method compared with the MPU method}

In order to show the stability of proposed method in the case of noise disturbance, we add different levels noise to the given data sampled from Stanford Bunny as shown in Fig. 7. Fig. 8 is the reconstructed Bunny from data points with different levels of noise by our proposed method. Fig. 9 exhibits the reconstructed Bunny with MPU method under various levels noise. It is seen that our proposed method is more robust to the noise than MPU method due to the strong stability of the radial basis functions. It can be seen from Fig. 9 that MPU mehod is completely
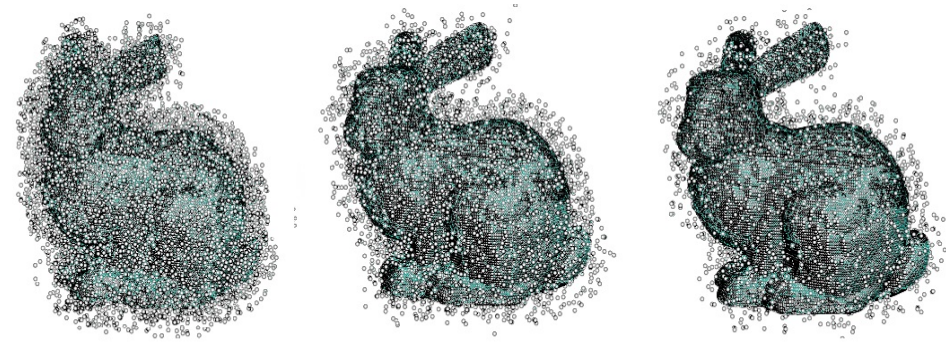

Fig. 7 Bunny data points with different levels of noise. 

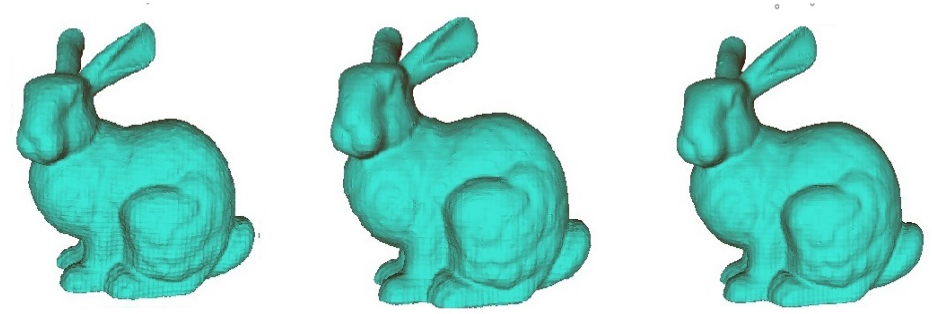

Fig. 8 Reconstructed Bunny with our proposed method from data points with different levels of noise.
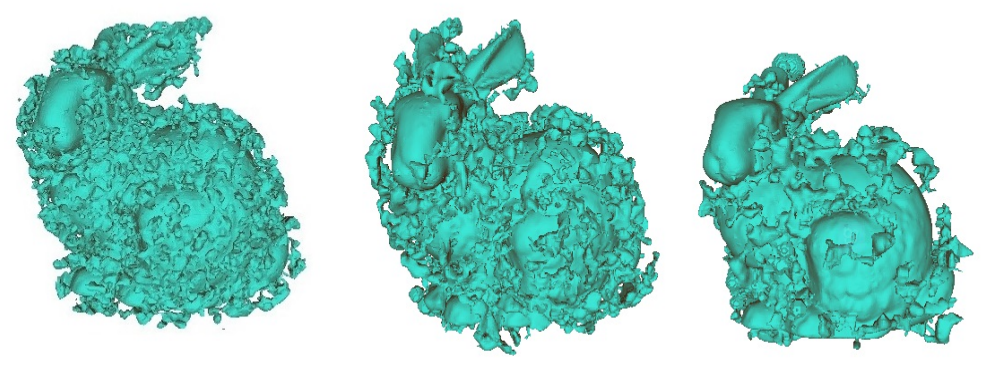

Fig. 9 Reconstructed Bunny with MPU method from data points with different levels of noise.

failed even if data is contaminated by low level of noise. Therefore, the proposed method can effectively resist noise disturbance and capture the shape of object.

\section{Conclusion}

This paper has presented a novel implicit surface reconstruction method based on partition of unity and local surface sheet approximation. Different to other surface reconstruction based on $\mathrm{RBF}$, our proposed method does not need to add offsurface points, reducing computational complexity. Furthermore, even in the case of noisy data, non-uniform data sampling, and objects with complex topological structure, Our method is capable of achieving satisfactory reconstruction results. So our method is quite effective and efficient in reconstructing a complex surface according to the given large datasets.

\section{Acknowledgement}

This work was supported by the Natural Science Foundation of Zhejiang Province of Cina (No. LSY19F020001, LY18F020018) 
Zhou Z., Fu Y., Zhao J.: An efficient method for surface reconstruction based on...

\section{References}

[1] ALEXA M., BEHR J., COHENOR D., FLEISHMANl S., Levin D. Silva CT Computing and rendering point set surfaces. IEEE Trans Vis Comput Graphics, 2003, 9(1), 3-15.

[2] Amenta N., CHOI S., KOlluRI R. The power crust. In: Proceedings 6rd Symp Solid Model Assoc Comput Mach, 2001, pp. 249-260.

[3] BLOOMENTHAL J. Polygonization of implicit surfaces. Comput Aided Geom Design, 1988, 5(4), pp. 341-355.

[4] BLOOMEnTHAL J., An implicit surface polygonizer. San Diego CA, USA: Academic Professional, Inc.,1994, pp. 324-349.

[5] CARR J.C., BEATSON R.K., CHERRIE J.B., MITCHELL T.J., FRIGHT W.R., MCCALLUM B.C., EVANS T.R. Reconstruction and representation of $3 \mathrm{~d}$ objects with radial basis functions. In: Proc 1rd of SIGGRAPH, 2001, pp. 67-76.

[6] DEY T.K., GOSWAMI S., Tight cocone: A water-tight surface reconstructor. J Computing Informat Sci Engin, 2003, 13, pp. 302-307.

[7] ZHOU Z.H., ZHAO J.W., CAO F.L. Surface reconstruction based on extreme learning machine. Neural Comput Appl, 2013, 23(2), pp. 283-292.

[8] EDELSBRUNNER H., MÜCKE E.P. Three-dimensional alpha shapes. ACM Trans Graphics (TOG), 1994, 13(1), pp. 43-72.

[9] SERGiO O.E., JOSE G.R., VICENTE M., CAZORLA M., PEREZ J.A.S., ALBERTO G.G. 3D surface reconstruction of noisy point clouds using growing neural gas: 3D object/scene reconstruction. Neural Process Let, 2016, 43(2), pp. 401-423.

[10] FORSEY D.R., BARTELS R.H. Hierarchical B-spline refinement. Comput Graphics, 1988, 22(4), pp. 205-212.

[11] HOPPE H., DEROSE T., DUChAMP T., MCDONALD J., STUETZLE W. Surface reconstruction from unorganized points. ACM SIGGRAPH92, 1992, pp. 71-78.

[12] KAZHDAN M., BOLITHO M., HOPPE H. Poisson surface reconstruction. In: Proc 4rd Eurographics Symp Geometry Processing, 2006, pp. 61-70.

[13] LAZZARO D., MONTEFUSCO L.B. Radial basis functions for the multivariate interpolation of large scattered data sets. In: Proc Computational Applied Math, 140'01, 2002, pp. 521536.

[14] LI Y., KIM J. Fast and efficient narrow volume reconstruction from scattered data. Pattern Recognit, 2015, 48(12), pp. 4057-4069.

[15] MACÊDO I., GOIS J.P., VELHO L. Hermite interpolation of implicit surfaces with radial basis functions. Brazilian Symp Comput Graphics Image Process, 2009, pp. 1-8.

[16] MACÊDO I., GOIS J.P., VELHO L. Hermite radial basis fuctions implicits. Comput Graphics Forum, 2011, 30(1), pp. 27-42.

[17] MACHUCHO-CADENA R., RIVERA-ROVELO J., BAYRO-CORROCHANO E. Geometric techniques for $3 \mathrm{D}$ tracking of ultrasound sensor, tumor segmentation in ultrasound images, and 3D reconstruction. Pattern Recogn, 2014, 47(5), pp. 1968-1987.

[18] NAGAI Y., OHTAKE Y., SUZUKI H., Smoothing of partition of unity implicit surfaces for noise robust surface reconstruction. Eurographics Symp Geometry Process, 2009, 28(5), pp. $1339-1348$.

[19] OHTAKE Y., BELYAEV A., ALEXEI M., TURK G., SEIDEL H.P. Multi-level partition of unity implicits. ACM Trans Graphics (TOG), 2003, 22(3), pp. 463-470.

[20] OHTAKE Y., BELYAEV A., ALEXEI M., TURK G., SEIDEL H.P. Multi-scale approach to $3 \mathrm{D}$ scattered data interplation with compactly supported basis functions. In: Proc Shape Model Inter, 2003.

[21] PARK I.K., LEE S.U., YUN I.D. Constructing NURBS surface model from scattered and unorganized range data. Inter Confer 3-D Imaging Model-3DIM, 1999, pp. 312-320.

[22] PAN R. J., MENG X. X., WHANGBO T. Hermite variational implicit surface reconstruction. Sci China Series F: Inform Sci, 2009, 52(2), pp. 308-315. 


\section{Neural Network World 3/2020, 161-176}

[23] RIDel B., GUennebaud G., ReUter P., GRAnIER X. Parabolic-cylindrical moving least squares surfaces. Comput Graphics, 2015, 51, pp. 60-66.

[24] TOBOR I., REUTER P., SCHLICK C. Efficient reconstructioin of large scattered geometric datasets using the partition of unity and radial basis functions. In: Proc WSCG, 2004.

[25] ZHAO H.K., OSHER S., MERRIMAN B., KANG M. Implicit and non-parametric shape reconstruction from unorganized points using variational level set method. Comput Vision Image Understanding, 2000, 80(3), pp. 295-319.

[26] ZHAO J.W., FU Y.Q., TAN Y.P., CAO F.L. A reduction algorithm for the big data in 3D surface reconstruction. In: Proc IEEE Intern Confer Systems, Man, Cybernetics, SMC2013, 2013, pp. 4843-4847. 